\title{
Erratum to: Optical temporal rogue waves in the generalized inhomogeneous nonlinear Schrödinger equation with varying higher-order even and odd terms
}

\author{
Yunqing Yang · Xin Wang · Zhenya Yan
}

Published online: 31 March 2015

(C) Springer Science+Business Media Dordrecht 2015

\section{Erratum to: Nonlinear Dyn \\ DOI 10.1007/s11071-015-2033-1}

There was an error in the first author's name in the original publication. The name is correct in this erratum.

The online version of the original article can be found under doi:10.1007/s11071-015-2033-1.

Y. Yang

School of Mathematics, Physics and Information Science,

Zhejiang Ocean University, Zhoushan 316004, China

Y. Yang $\cdot$ Z. Yan $(\varangle)$

Key Laboratory of Mathematics Mechanization, Institute of Systems Science, AMSS,

Chinese Academy of Sciences, Beijing 100190, China

e-mail: zyyan@mmrc.iss.ac.cn

X. Wang

Shanghai Key Laboratory of Trustworthy Computing, East China Normal University, Shanghai 200062, China 